\title{
Laguerre approach for solving system of linear Fredholm integro-differential equations
}

\author{
Zaffer Elahi ${ }^{1} \cdot$ Ghazala Akram $^{1} \cdot$ Shahid S. Siddiqi ${ }^{2}$
}

Received: 30 January 2018/Accepted: 27 July 2018/Published online: 10 August 2018

(C) The Author(s) 2018

\begin{abstract}
A numerical scheme has been developed for solving the system of linear Fredholm integro-differential equations subject to the mixed conditions using Laguerre polynomials. Using collocation method, the system of Fredholm integro-differential equations has been transformed to the system of linear equations in unknown Laguerre coefficients, which leads to the solution in terms of Laguerre polynomials. Moreover, the accuracy and applicability of the scheme have been compared with Tau method and Adomian decomposition method that reveals the proposed scheme to be more efficient.
\end{abstract}

Keywords System of Fredholm integro-differential equations · Laguerre polynomials · Collocation method

Mathematics Subject Classification 45A05 - 45J05

\section{Introduction}

There are many branches of science, such as control theory and financial mathematics, which leads to integro-differential equations (IDEs). In modern mathematics, IDEs mostly occur in many applied areas including engineering, physics and biology [1-6]. The resolution of many problems in physics and engineering leads to differential and integral equations in bounded or unbounded domains. For example, problems occur in coastal hydrodynamics and in meteorology. The integrals appear in many physical contexts, containing the product of orthogonal polynomials or special functions. For example, the wave functions of the hydrogen as well as 2-, 3- and, in general, $n$-dimensional harmonic oscillator encompassing Laguerre polynomials

Ghazala Akram

toghazala2003@yahoo.com

Zaffer Elahi

zafferelahi@gmail.com

Shahid S. Siddiqi

shahidsiddiqiprof@yahoo.co.uk

1 Department of Mathematics, University of the Punjab, Quaide-Azam Campus, Lahore 54590, Pakistan

2 Faculty of Information and Technology, University of Central Punjab, 1-Khayaban-e-Jinnah Road, Johar Town, Lahore 54770, Pakistan and the evaluation of integrals having the product of these polynomials are essential [7].

In the fields of applied mathematics and scientific computing, spectral methods [8-10] became popular among researchers as a robust numerical tool. The remarkable results are obtained, using the spectral methods, to solve the problems [11-13] in different fields of natural sciences. Moreover, system of IDEs found in the field of science and engineering, such as nano-hydrodynamics [14], glass-forming process [15], dropwise condensation [16], wind ripples in the desert [17], modeling the competition between tumor cell and the immune system [18] and examining the noise term phenomenon [19, 20]. Since analytical solutions of such type of problems are hard to determine, therefore the numerical methods are required. Many researchers presented numerical methods for system of IDEs, for instance the Tau method [21], Fibonacci matrix method [22], Bessel matrix method [23, 24], Adomian decomposition method (ADM) [25], modified decomposition method [26], Galerkin methods with hybrid functions [27-30, 38], differential transform method [31] and the block pulse functions method [32].

The main objective of this paper is to study the concept of the system of IDEs and manipulate the Laguerre matrix method for solving the system of linear Fredholm IDEs.

The following system of linear Fredholm IDEs has been considered 


$$
\begin{array}{r}
\sum_{k=0}^{n} \sum_{q=1}^{r} \rho_{p, q}^{k}(s) u_{q}^{(k)}(s)=f_{p}(s)+\int_{a}^{b} \sum_{q=1}^{r} K_{p, q}(s, t) u_{q}(t) \mathrm{d} t \\
p=1,2, \ldots, r, \quad 0 \leq a \leq s \leq b
\end{array}
$$

subject to

\section{Matrix relation}

The matrix form of Laguerre polynomials $L_{n}(s)$ is as follows

$$
\mathbf{L}^{\mathrm{T}}(s)=\mathbf{H} \mathbf{S}^{\mathrm{T}}(s) \Leftrightarrow \mathbf{L}(s)=\mathbf{S}(s) \mathbf{H}^{\mathrm{T}},
$$

where

$$
\begin{aligned}
\mathbf{L}(s) & =\left(\begin{array}{lllll}
L_{0}(s) & L_{1}(s) & L_{2}(s) & \ldots & L_{N}(s)
\end{array}\right), \\
\mathbf{S}(s) & =\left(\begin{array}{llcll}
1 & s & s^{2} & \ldots & s^{N}
\end{array}\right),
\end{aligned}
$$

and

$$
\begin{aligned}
& \sum_{q=0}^{n-1}\left(a_{p, q}^{k} u_{k}^{(q)}(a)+b_{p, q}^{k} u_{k}^{(q)}(b)\right)=\mu_{k, p}, \quad p=0,1, \ldots, n-1, \\
& \quad k=1,2, \ldots, r
\end{aligned}
$$

where $u_{q}(s)$ is the unknown and $\rho_{p, q}^{k}, K_{p, q}(s, t), f_{p}(s)$ are the known functions defined in the interval $[a, b]$, the kernel function $K_{p, q}(s, t)$ can be expanded using Maclaurin series and also $a_{p, q}^{k}, b_{p, q}^{k}, \mu_{k, p}$ are real constants.

Taking $u_{p}(s)$ to be the approximate solution of Eq. (1) in terms of truncated Laguerre series yields

$$
u_{p}(s)=\sum_{n=0}^{N} \alpha_{p, n} L_{n}(s), \quad p=1,2, \ldots, r, \quad a \leq s \leq b,
$$

where $\alpha_{p, n}$ are the unknown Laguerre coefficients, to be determined for $n=0,1,2, \ldots, N$, and $L_{n}(s)$ be the Laguerre polynomial defined by

$L_{n}(s)=\sum_{k=0}^{n} \frac{(-1)^{k}}{k !}\left(\begin{array}{c}n \\ n-k\end{array}\right) s^{k}, \quad 0 \leq s \leq b<\infty$.
The relation defined in Eq. (3) can be written in matrix form, as

$\left(u_{q}(s)\right)=\mathbf{L}(\mathbf{s}) \mathbf{A}_{\mathbf{q}}, \quad \mathbf{q}=\mathbf{1}, \mathbf{2}, \ldots, \mathbf{r}$,

where

$\mathbf{A}_{q}=\left(\alpha_{q, 0}, \alpha_{q, 1}, \ldots, \alpha_{q, N}\right)^{\mathrm{T}}$

or from Eq. (4)

$\left(u_{q}(s)\right)=\mathbf{S}(\mathbf{s}) \mathbf{H}^{\mathrm{T}} \mathbf{A}_{\mathbf{q}}, \quad \mathbf{q}=\mathbf{1}, \mathbf{2}, \ldots, \mathbf{r}$.

Also, the relation between the matrix $\mathbf{S}(s)$ and its first derivative is

$\mathbf{S}^{(1)}(s)=S(s) \mathbf{B}, \quad \mathbf{S}^{(0)}(s)=\mathbf{S}(s)$,

where

$\mathbf{B}=\left(\begin{array}{ccccc}0 & 1 & 0 & \ldots & 0 \\ 0 & 0 & 2 & \ldots & 0 \\ \vdots & \vdots & \vdots & \ddots & \vdots \\ 0 & 0 & 0 & \ldots & N \\ 0 & 0 & 0 & \ldots & 0\end{array}\right)$.

It follows from Eqs. (4) and (6) that 
$\mathbf{S}^{(p)}(s)=\mathbf{S}^{(p-1)}(s) \mathbf{B}=\mathbf{S}(s)(\mathbf{B})^{p}, \quad p=0,1,2, \ldots, n$,

and thus

$\mathbf{L}^{(p)}(s)=\mathbf{S}^{(p)}(s) \mathbf{H}^{\mathrm{T}}=\mathbf{S}(s)(\mathbf{B})^{p} \mathbf{H}^{\mathrm{T}}, \quad p=0,1,2, \ldots, n$.

The following recurrence relation is obtained using Eqs. (5), (7) and (8)

$$
\begin{aligned}
u_{q}^{(p)}(s) & =\mathbf{L}^{(p)}(s) \mathbf{A}_{q} \\
& =\mathbf{S}^{(p)}(s) \mathbf{H}^{\mathrm{T}} \mathbf{A}_{q} \\
& =\mathbf{S}(s)(\mathbf{B})^{p} \mathbf{H}^{\mathrm{T}} \mathbf{A}_{q}, \quad p=0,1,2, \ldots, n, q=1,2, \ldots, r .
\end{aligned}
$$

Hence, the matrices $\mathbf{u}^{(p)}(s)$ can be expressed as

$\mathbf{u}^{(p)}(s)=\overline{\mathbf{S}}(s)(\overline{\mathbf{B}})^{p} \overline{\mathbf{H}} \mathbf{A}, \quad p=0,1,2, \ldots, n$,

where

$\mathbf{u}^{(p)}(s)=\left(\begin{array}{c}u_{1}^{(p)}(s) \\ u_{2}^{(p)}(s) \\ \vdots \\ u_{r}^{(p)}(s)\end{array}\right), \quad \mathbf{A}=\left(\begin{array}{c}\mathbf{A}_{1} \\ \mathbf{A}_{2} \\ \vdots \\ \mathbf{A}_{r}\end{array}\right)$,

$\overline{\mathbf{S}}(s)=\left(\begin{array}{cccc}\mathbf{S}(s) & 0 & \ldots & 0 \\ 0 & \mathbf{S}(s) & \ldots & 0 \\ \vdots & \vdots & \ddots & \vdots \\ 0 & 0 & \ldots & \mathbf{S}(s)\end{array}\right)_{r \times r}$,

$\overline{\mathbf{B}}=\left(\begin{array}{cccc}\mathbf{B} & 0 & \cdots & 0 \\ 0 & \mathbf{B} & \cdots & 0 \\ \vdots & \vdots & \ddots & \vdots \\ 0 & 0 & \cdots & \mathbf{B}\end{array}\right)_{r \times r}$ and

$\overline{\mathbf{H}}=\left(\begin{array}{cccc}\mathbf{H}^{\mathrm{T}} & 0 & \cdots & 0 \\ 0 & \mathbf{H}^{\mathrm{T}} & \cdots & 0 \\ \vdots & \vdots & \ddots & \vdots \\ 0 & 0 & \cdots & \mathbf{H}^{\mathrm{T}}\end{array}\right)$.

\section{Method of solution}

The matrix form of the system defined in Eq. (1) is as follows

$\sum_{p=0}^{n} \mathbf{P}_{p}(s) \mathbf{u}^{(p)}(s)=\boldsymbol{f}(s)+\mathbf{I}(s)$

where $\mathbf{u}^{(p)}(s)=\left(\begin{array}{c}u_{1}^{(p)}(s) \\ u_{2}^{(p)}(s) \\ \vdots \\ u_{r}^{(p)}(s)\end{array}\right), \quad \boldsymbol{f}=\left(\begin{array}{c}f_{1}(s) \\ f_{2}(s) \\ \vdots \\ f_{r}(s)\end{array}\right)$

$\mathbf{P}_{p}(s)=\left(\begin{array}{cccc}\rho_{1,1}^{p}(s) & \rho_{1,2}^{p}(s) & \ldots & \rho_{1, r}^{p}(s) \\ \rho_{2,1}^{p}(s) & \rho_{2,2}^{p}(s) & \ldots & \rho_{2, r}^{p}(s) \\ \vdots & \vdots & \ddots & \vdots \\ \rho_{r, 1}^{p}(s) & \rho_{r, 2}^{p}(s) & \ldots & \rho_{r, r}^{p}(s)\end{array}\right)$,

$\mathbf{I}(s)=\int_{a}^{b} \mathbf{K}(s, t) u(t) \mathrm{d} t$,

$\mathbf{K}(s, t)=\left(\begin{array}{cccc}K_{1,1}(s, t) & K_{1,2}(s, t) & \ldots & K_{1, r}(s, t) \\ K_{2,1}(s, t) & K_{2,2}(s, t) & \ldots & K_{2, r}(s, t) \\ \vdots & \vdots & \ddots & \vdots \\ K_{r, 1}(s, t) & K_{r, 2}(s, t) & \ldots & K_{r, r}(s, t)\end{array}\right)$,

$\mathbf{I}=\left(\begin{array}{c}I_{1}(s) \\ I_{2}(s) \\ \vdots \\ I_{r}(s)\end{array}\right)$

and

$I_{p}(s)=\int_{a}^{b} \sum_{q=1}^{r} K_{p, q}(s, t) u_{q}(t)$.

Using truncated Taylor and Laguerre series to approximate the kernel $K_{p, q}(s, t)$ yields

$$
\begin{aligned}
K_{p, q}(s, t) & =\sum_{m=0}^{N} \sum_{n=0}^{N} k_{p q}^{t} s^{m} t^{n} \quad \text { and } \\
K_{p, q}(s, t) & =\sum_{m=0}^{N} \sum_{n=0}^{N} k_{p q}^{l} L_{m}(s) L_{n}(t),
\end{aligned}
$$

where

$$
\begin{gathered}
k_{p q}^{t}=\frac{1}{m ! n !} \frac{\partial^{m+n} K(0,0)}{\partial s^{m} \partial t^{n}}, \quad m, n=0,1,2, \ldots, N, \\
p, q=0,1,2, \ldots, r .
\end{gathered}
$$

Equation (13) can be put into the matrix form, as

$$
K_{p, q}(s, t)=\mathbf{S}(s) \mathbf{K}_{p q}^{t} \mathbf{S}^{\mathrm{T}}(t), \quad \mathbf{K}_{p q}^{t}=\left(k_{p q}^{t}\right),
$$

and

$$
K_{p, q}(s, t)=\mathbf{L}(s) \mathbf{K}_{p q}^{l} \mathbf{L}^{\mathrm{T}}(t), \quad \mathbf{K}_{p q}^{l}=\left(k_{p q}^{l}\right) .
$$

Using Eqs. (5) and (15) in Eq. (12) to get the matrix form of the integral part yields 


$$
\begin{aligned}
\left(I_{p}(s)\right) & =\int_{a}^{b} \sum_{q=0}^{r} \mathbf{L}(s) \mathbf{K}_{p q}^{l} \mathbf{L}^{\mathrm{T}}(t) \mathbf{S}(t) \mathbf{H}^{\mathrm{T}} \mathbf{A}_{q} \mathrm{~d} t \\
& \left.=\sum_{q=0}^{r} \int_{a}^{b} \mathbf{L}(s) \mathbf{K}_{p q}^{l} \mathbf{L}^{(} T\right)(t) \mathbf{S}(t) \mathbf{H}^{\mathrm{T}} \mathbf{A}_{q} \mathrm{~d} t \\
& =\sum_{q=0}^{r} \mathbf{L}(s) \mathbf{K}_{p q}^{l} \mathbf{Q} \mathbf{A}_{q}
\end{aligned}
$$

$\mathbf{P}_{p}=\left(\begin{array}{cccc}\mathbf{P}_{p}\left(s_{0}\right) & 0 & \ldots & 0 \\ 0 & \mathbf{P}_{p}\left(s_{1}\right) & \ldots & 0 \\ \vdots & \vdots & \ddots & \vdots \\ 0 & 0 & \ldots & \mathbf{P}_{p}\left(s_{N}\right)\end{array}\right)$

$\mathbf{U}^{(p)}=\left(\begin{array}{c}\mathbf{u}^{(p)}\left(s_{0}\right) \\ \mathbf{u}^{(p)}\left(s_{1}\right) \\ \vdots \\ \mathbf{u}^{(p)}\left(s_{N}\right)\end{array}\right), \quad \boldsymbol{F}=\left(\begin{array}{c}\boldsymbol{f}\left(s_{0}\right) \\ \boldsymbol{f}\left(s_{1}\right) \\ \vdots \\ \boldsymbol{f}\left(s_{N}\right)\end{array}\right)$

$$
\begin{aligned}
\mathbf{Q} & =\int_{a}^{b} \mathbf{L}^{\mathrm{T}}(t) \mathbf{S}(t) \mathbf{H}^{\mathrm{T}} \mathrm{d} t \\
& =\int_{a}^{b} \mathbf{H S}^{\mathrm{T}}(t) \mathbf{S}(t) \mathbf{H}^{\mathrm{T}} \mathrm{d} t=\mathbf{H} \mathbf{M} \mathbf{H}^{\mathrm{T}},
\end{aligned}
$$

and $\mathbf{I}=\left(\begin{array}{c}\mathbf{I}\left(s_{0}\right) \\ \mathbf{I}\left(s_{1}\right) \\ \vdots \\ \mathbf{I}\left(s_{N}\right)\end{array}\right)$

where

$$
\begin{gathered}
\mathbf{M}=\int_{a}^{b} \mathbf{S}^{\mathrm{T}}(t) \mathbf{S}(t) \mathrm{d} t=\left(m_{i j}\right), \quad m_{i j}=\frac{b^{i+j+1}-a^{i+j+1}}{i+j+1}, \\
i, j=0,1,2, \ldots, N .
\end{gathered}
$$

Using the collocation points in Eq. (10) yields

$$
\begin{gathered}
\mathbf{u}^{(p)}\left(s_{t}\right)=\overline{\mathbf{S}}\left(s_{t}\right)(\overline{\mathbf{B}})^{p} \overline{\mathbf{H}} \mathbf{A}, \quad t=0,1,2, \ldots, N, \\
p=0,1,2, \ldots, r,
\end{gathered}
$$

Using Eq. (4) in Eq. (16) leads to

$$
\left(I_{p}(s)\right)=\sum_{q=0}^{r} \mathbf{S}(s) \mathbf{H}^{\mathrm{T}} \mathbf{K}_{p q}^{l} \mathbf{Q} \mathbf{A}_{q} .
$$

In order to determine the system of equations in matrix form, replacing the collocation points defined by

$s_{t}=a+\frac{b-a}{N} t, \quad t=0,1,2, \ldots, N$,

in Eq. (11), leads to

$\sum_{p=0}^{n} \mathbf{P}_{p}\left(s_{t}\right) \mathbf{u}^{(p)}\left(s_{t}\right)=\boldsymbol{f}\left(s_{t}\right)+\mathbf{I}\left(s_{t}\right)$

or

$\sum_{p=0}^{n} \mathbf{P}_{p} \mathbf{U}^{(p)}=\boldsymbol{F}+\mathbf{I}$

where

which can be written as

$\mathbf{U}^{(p)}=\mathbf{S}(\overline{\mathbf{B}})^{p} \overline{\mathbf{H}} \mathbf{A}$

where

$\mathbf{S}=\left(\begin{array}{c}\overline{\mathbf{S}}\left(s_{0}\right) \\ \overline{\mathbf{S}}\left(s_{1}\right) \\ \vdots \\ \overline{\mathbf{S}}\left(s_{N}\right)\end{array}\right)$ and

$\overline{\mathbf{S}}\left(s_{t}\right)=\left(\begin{array}{cccc}\mathbf{S}\left(s_{t}\right) & 0 & \ldots & 0 \\ 0 & \mathbf{S}\left(s_{t}\right) & \ldots & 0 \\ \vdots & \vdots & \ddots & \vdots \\ 0 & 0 & \ldots & \mathbf{S}\left(s_{t}\right)\end{array}\right)_{r \times r}, \begin{aligned} & t=0,1,2, \ldots, N, \\ & p=0,1,2, \ldots, r .\end{aligned}$

Substituting the collocation points in Eq. (17) leads to

$$
\begin{gathered}
\left(I_{p}\left(s_{t}\right)\right)=\sum_{q=0}^{r} \mathbf{S}\left(s_{t}\right) \mathbf{H}^{\mathrm{T}} \mathbf{K}_{p q}^{l} \mathbf{Q} \mathbf{A}_{q}, \quad t=0,1,2, \ldots, N, \\
p=0,1,2, \ldots, r .
\end{gathered}
$$

Similarly, substituting the collocation points into the matrix $\mathbf{I}(s)$ of Eq. (11) and using Eq. (21) yields

$$
\mathbf{I}\left(s_{t}\right)=\overline{\mathbf{S}}\left(s_{t}\right) \overline{\mathbf{H}} \mathbf{K}_{l} \overline{\mathbf{Q}} \mathbf{A}, t=0,1,2, \ldots, N, p=0,1,2, \ldots, r,
$$

where 
$\mathbf{I}\left(s_{t}\right)=\left(\begin{array}{c}I_{1}\left(s_{t}\right) \\ I_{2}\left(s_{t}\right) \\ \vdots \\ I_{r}\left(s_{t}\right)\end{array}\right)$

$\overline{\mathbf{S}}\left(s_{t}\right)=\left(\begin{array}{cccc}\mathbf{S}\left(s_{t}\right) & 0 & \ldots & 0 \\ 0 & \mathbf{S}\left(s_{t}\right) & \ldots & 0 \\ \vdots & \vdots & \ddots & \vdots \\ 0 & 0 & \ldots & \mathbf{S}\left(s_{t}\right)\end{array}\right)_{r \times r}$,

$\overline{\mathbf{H}}=\left(\begin{array}{cccc}\mathbf{H}^{\mathrm{T}} & 0 & \cdots & 0 \\ 0 & \mathbf{H}^{\mathrm{T}} & \cdots & 0 \\ \vdots & \vdots & \ddots & \vdots \\ 0 & 0 & \cdots & \mathbf{H}^{\mathrm{T}}\end{array}\right)$

$\mathbf{K}_{l}=\left(\begin{array}{cccc}\mathbf{K}_{11}^{l} & \mathbf{K}_{12}^{l} & \ldots & \mathbf{K}_{1 r}^{l} \\ \mathbf{K}_{21}^{l} & \mathbf{K}_{22}^{l} & \ldots & \mathbf{K}_{2 r}^{l} \\ \vdots & \vdots & \ddots & \vdots \\ \mathbf{K}_{r 1}^{l} & \mathbf{K}_{r 2}^{l} & \ldots & \mathbf{K}_{r r}^{l}\end{array}\right)$,

$\overline{\mathbf{Q}}=\left(\begin{array}{cccc}\mathbf{Q} & 0 & \ldots & 0 \\ 0 & \mathbf{Q} & \ldots & 0 \\ \vdots & \vdots & \ddots & \vdots \\ 0 & 0 & \ldots & \mathbf{Q}\end{array}\right) \quad$ and $\quad \mathbf{A}=\left(\begin{array}{c}\mathbf{A}_{1} \\ \mathbf{A}_{2} \\ \vdots \\ \mathbf{A}_{r}\end{array}\right)$.

Thus, using Eq. (22) in I defined in Eq. (19) gives

$\mathbf{I}=\left(\begin{array}{c}\mathbf{I}\left(s_{0}\right) \\ \mathbf{I}\left(s_{1}\right) \\ \vdots \\ \mathbf{I}\left(s_{N}\right)\end{array}\right)=\mathbf{S} \overline{\mathbf{H}} \mathbf{K}_{l} \overline{\mathbf{Q}} \mathbf{A}$.

Hence, from Eq. (19) the fundamental matrix equation of the system defined in Eq. (1), using Eqs. (20) and (23), is obtained as under

$\left[\sum_{p=0}^{n} \mathbf{P}_{p} \mathbf{S}(\overline{\mathbf{B}})^{p} \overline{\mathbf{H}}-\mathbf{S} \overline{\mathbf{H}} \mathbf{K}_{l} \overline{\mathbf{Q}}\right] \mathbf{A}=\boldsymbol{F}$.

$r(N+1) \times r(N+1)$ and $r(N+1) \times 1$ are the dimensions of the respective matrices $\mathbf{P}_{p}, \mathbf{S},(\overline{\mathbf{B}})^{p}, \overline{\mathbf{H}}, \mathbf{K}_{l}, \overline{\mathbf{Q}}$ and $\mathbf{A}, \boldsymbol{F}$. Moreover, Eq. (24) is written in more instructive form, as
$\mathbf{W}=\left(w_{m, n}\right)=\sum_{p=0}^{n} \mathbf{P} p \mathbf{S}(\overline{\mathbf{B}})^{p} \overline{\mathbf{H}}-\mathbf{S} \overline{\mathbf{H}} \mathbf{K}_{l} \overline{\mathbf{Q}}, m, n=1,2, \ldots, r(N+1)$.

The conditions defined in Eq. (2) can be expressed in the following matrix form

$\sum_{q=0}^{n-1}\left[a_{p, q}^{1} u_{1}^{(q)}(a)+b_{p, q}^{1} u_{1}^{(q)}(b)\right]=\mu_{1, p}$,
$\sum_{q=0}^{n-1}\left[a_{p, q}^{2} u_{2}^{(q)}(a)+b_{p, q}^{2} u_{2}^{(q)}(b)\right]=\mu_{2, p}$,

$\sum_{q=0}^{n-1}\left[a_{p, q}^{r} u_{r}^{(q)}(a)+b_{p, q}^{r} u_{r}^{(q)}(b)\right]=\mu_{r, p}$

or

$\sum_{q=0}^{n-1}\left[a_{q}^{1} u_{1}^{(q)}(a)+b_{q}^{1} u_{1}^{(q)}(b)\right]=\mu_{1}$

$\sum_{q=0}^{n-1}\left[a_{q}^{2} u_{2}^{(q)}(a)+b_{q}^{2} u_{2}^{(q)}(b]=\mu_{2}\right.$,

$\sum_{q=0}^{n-1}\left[a_{q}^{r} u_{r}^{(q)}(a)+b_{q}^{r} u_{r}^{(q)}(b)\right]=\mu_{r}$

where

$\mu_{p}=\left(\begin{array}{c}\mu_{p, 0} \\ \mu_{p, 1} \\ \vdots \\ \mu_{p, n-1}\end{array}\right)_{n \times 1}, \quad a_{q}^{p}=\left(\begin{array}{c}a_{0, q}^{p} \\ a_{1, q}^{p} \\ \vdots \\ a_{n-1, q}^{p}\end{array}\right)_{n \times 1}$,

$b_{q}^{p}=\left(\begin{array}{c}b_{0, q}^{p} \\ b_{1, q}^{p} \\ \vdots \\ b_{n-1, q}^{p}\end{array}\right)_{n \times 1}, \quad p=1,2, \ldots, r$.

or briefly

$\sum_{q=0}^{n-1}\left[a_{q} u^{(q)}(a)+b_{q} u^{(q)}(b)\right]=\mu$,

where
$\mathbf{W A}=\boldsymbol{F}, \quad$ or $\quad(\mathbf{W} ; \boldsymbol{F})$,

where 
$\begin{aligned} a_{q}= & \left(\begin{array}{cccc}a_{q}^{1} & 0 & \ldots & 0 \\ 0 & a_{q}^{2} & \ldots & 0 \\ \vdots & \vdots & \ddots & \vdots \\ 0 & 0 & \ldots & a_{q}^{r}\end{array}\right)_{r \times r}, \\ b_{q}= & \left(\begin{array}{cccc}b_{q}^{1} & 0 & \ldots & 0 \\ 0 & b_{q}^{2} & \ldots & 0 \\ \vdots & \vdots & \ddots & \vdots \\ 0 & 0 & \ldots & b_{q}^{r}\end{array}\right)_{r \times r} \text { and } \mu=\left(\begin{array}{c}\mu_{1} \\ \mu_{2} \\ \vdots \\ \mu_{r}\end{array}\right)_{r \times 1} .\end{aligned}$

Putting the values of $u^{(q)}(a)$ and $u^{(q)}(b)$ from Eq. (10) into Eq. (26) yields

$\sum_{q=0}^{n-1}\left[a_{q} \overline{\mathbf{S}}(a)+b_{q} \overline{\mathbf{S}}(b)\right](\overline{\mathbf{B}})^{q} \overline{\mathbf{H}} \mathbf{A}=\mu$.

or briefly

$\mathbf{V A}=\mu$ or $(\mathbf{V} ; \mu)$,

where

$\mathbf{V}=\sum_{q=0}^{n-1}\left[a_{q} \overline{\mathbf{S}}(a)+b_{q} \overline{\mathbf{S}}(b)\right](\overline{\mathbf{B}})^{q} \overline{\mathbf{H}}$

Thus, Eq. (28) is the matrix form of the conditions defined in Eq. (2). Replacing the augmented matrix in Eq. (28) with the augmented matrix in Eq. (25) yields

$\tilde{\mathbf{W}} \mathbf{A}=\tilde{\boldsymbol{F}}$.

By replacing the $n r$-rows of the matrix $\mathbf{W}$, the augmented matrix of the above system can be obtained as under [23, 33-36]
It is not necessary to replace the last rows of $\mathbf{W}$. For instance, if the matrix $\mathbf{W}$ is singular, then the rows that are linearly dependent (or have same factors) or all zeros are replaced. If $\operatorname{rank} \tilde{\mathbf{W}}=\operatorname{rank} \quad(\tilde{\mathbf{W}} ; \tilde{\boldsymbol{F}})=r(N+1)$, then $\mathbf{A}=(\tilde{\mathbf{W}})^{-1} \tilde{\boldsymbol{F}}$. Thus, $u_{p}(s)$ can be approximated by Eq. (3). However, if $|\tilde{W}|=0$, then a particular solution may be found; otherwise, the solution will not be possible.

\section{Error analysis}

Since Eq. (3) approximates the system defined in Eq. (1), therefore substituting $u_{q}(s)$ by $u_{p, N}(s), p=1,2, \ldots, r$ in Eq. (1), the resulting equation must be satisfied approximately, i.e., for $s=s_{i} \in[a, b], i=0,1,2, \ldots$.

$$
\begin{aligned}
E_{p}\left(s_{i}\right)= & \mid \sum_{k=0}^{n} \sum_{q=1}^{r} \rho_{p, q}^{k}\left(s_{i}\right) u_{p, N}^{(k)}\left(s_{i}\right)-f_{p}\left(s_{i}\right) \\
& -\int_{a}^{b} \sum_{q=1}^{r} K_{p, q}\left(s_{i}, t\right) u_{p, N}(t) \mathrm{d} t \mid \cong 0, p=1,2, \ldots, r
\end{aligned}
$$

and $E_{p}\left(s_{i}\right) \leq 10^{-k_{i}}$, where $k_{i}$ is a nonnegative integer. If $\max 10^{-k_{i}}=10^{-k}$ ( $k$ positive integer) is prescribed, then the truncation limit $N$ is increased till the difference $E_{p}\left(s_{i}\right)$ at each of the points becomes smaller than the prescribed $10^{-k}[23,36,37]$. For $\max 10^{-k_{i}} \neq 10^{-k}$, the error can be estimated by the following function

$$
(\tilde{\mathbf{W}} ; \tilde{\boldsymbol{F}})=\left(\begin{array}{cccccc}
w_{1,1} & w_{1,2} & \ldots & w_{1, r(N+1)} & ; & f_{1}\left(s_{0}\right) \\
\vdots & \vdots & \ddots & \vdots & ; & \vdots \\
w_{r, 1} & w_{r, 2} & \ldots & w_{r, r(N+1)} & ; & f_{r}\left(s_{0}\right) \\
\vdots & \vdots & \ddots & \vdots & ; & \vdots \\
w_{r(N-n+1), 1} & w_{r(N-n+1), 2} & \ldots & w_{r(N-n+1), r(N+1)} & ; & f_{r}\left(s_{N-n}\right) \\
v_{1,1} & v_{1,2} & \ldots & v_{1, r(N+1)} & ; & \mu_{1,0} \\
\vdots & \vdots & \ddots & \vdots & ; & \vdots \\
v_{r, 1} & v_{r, 2} & \vdots & v_{r, r(N+1)} & ; & \mu_{1, n-1} \\
\vdots & \vdots & \ddots & \vdots & ; & \vdots \\
v_{n r, 1} & v_{n r, 2} & \cdots & v_{n r, r(N+1)} & ; & \mu_{r, n-1}
\end{array}\right) .
$$




$$
\begin{aligned}
E_{p, N}(s)= & \sum_{k=0}^{n} \sum_{q=1}^{r} \rho_{p, q}^{k}(s) u_{q}^{(k)}(s)-f_{p}(s) \\
& -\int_{a}^{b} \sum_{q=1}^{r} K_{p, q}(s, t) u_{q}(t) \mathrm{d} t \cong 0, \quad p=1,2, \ldots, r .
\end{aligned}
$$

The error will be decreasing, if $E_{p, N}(s) \rightarrow 0$, for sufficiently large $N$.

\section{Numerical examples}

Following examples have been considered to examine the reliability and efficiency of the proposed technique.

Example 1 Consider the system of Fredholm IDEs, as

$u_{1}^{(2)}(s)+u_{2}^{(1)}(s)=3 s^{2}+\frac{3 s}{10}+8-\int_{0}^{1} 2 s t\left\{u_{1}(t)-3 u_{2}(t)\right\} \mathrm{d} t$

$u_{1}^{(1)}(s)+u_{2}^{(2)}(s)=21 s+\frac{4}{5}-\int_{0}^{1} 3\left(2 s+t^{2}\right)\left\{u_{1}(t)-2 u_{2}(t)\right\} \mathrm{d} t$

$$
0 \leq s \leq 1
$$

subject to the following mixed conditions

$$
\begin{gathered}
u_{1}(0)+u_{1}^{(1)}(0)=1, \quad u_{1}(1)+u_{1}^{(1)}(1)=10 \\
u_{2}(0)+u_{2}^{(1)}(0)=1, \quad u_{2}(1)+u_{2}^{(1)}(1)=7 .
\end{gathered}
$$

The analytical solutions are $u_{1}(s)=3 s^{2}+1$ and $u_{2}(s)=s^{3}+2 s-1$.

Solving the system of equations for $N=3$ by following the procedure stated above yields the approximate solutions $\quad u_{1,3}(s)=3 s^{2}+1 \quad$ and $\quad u_{2,3}(s)=s^{3}+2 s-1$ which are exactly the same as the analytical one. Table 1 shows the numerical results obtained by the proposed technique and their comparison with Tau method [21], whereas Figs. 1 and 2 depict the absolute errors $e_{1,3}$ and $e_{2,3}$ at $N=3$ for Example 1.

Example 2 Consider the system of Fredholm IDEs, as

$$
\begin{aligned}
& u_{1}^{(2)}(s)=\frac{8}{9}+\int_{0}^{1}\left\{\frac{1}{3} u_{1}(t)+\frac{1}{4} u_{2}(t)\right\} \mathrm{d} t \\
& u_{2}^{(2)}(s)=6 s-\frac{s^{2}}{18}+\int_{0}^{1}\left\{\frac{s^{2}}{6} u_{1}(t)-\frac{s^{2}}{3} u_{2}(t)\right\} \mathrm{d} t, \quad 0 \leq s \leq 1,
\end{aligned}
$$

subject to the following initial conditions

$$
u_{1}(0)=0, \quad u_{1}^{(1)}(0)=\frac{1}{3}, \quad u_{2}(0)=0, \quad u_{2}^{(1)}(0)=-\frac{1}{2} .
$$

The analytical solution is $u_{1}(s)=\frac{s^{2}}{2}+\frac{s}{3}$ and $u_{2}(s)=s^{3}-\frac{s}{2}$.

Solving the system of equations for $N=3$ by following the procedure stated above yields the approximate solutions $u_{1,3}(s)=\frac{s^{2}}{2}+\frac{s}{3}$ and $u_{2,3}(s)=s^{3}-\frac{s}{2}$ which are exactly the same as the analytical one. Numerical results obtained by the proposed technique are shown in Table 2, while the comparison of the maximum absolute errors of the proposed technique with Adomian decomposition method [25] is $\quad\left\|e\left(u_{1,3}\right)\right\|_{\infty}=0, \quad\left\|e\left(u_{2,3}\right)\right\|_{\infty}=0 \quad$ and $\left\|e\left(u_{1,6}\right)\right\|_{\infty}=0.2 \times 10^{-6},\left\|e\left(u_{2,6}\right)\right\|_{\infty}=0$, respectively.

Example 3 Consider the system of Fredholm IDEs, as

Table 1 Numerical results for Example 1

\begin{tabular}{lllll}
\hline$s$ & $\begin{array}{l}\text { Exact } \\
u_{1}(s)\end{array}$ & $\begin{array}{l}\text { Proposed } \\
u_{1,3}(s)\end{array}$ & $\begin{array}{l}\text { Absolute errors } \\
e_{1,3}(s)\end{array}$ & $\begin{array}{l}\text { Absolute errors } \\
\text { Tau method }[21]\end{array}$ \\
\hline 0 & 1 & 1 & 0 & $3 \times 10^{-14}$ \\
0.2 & 1.12 & 1.12 & 0 & $2 \times 10^{-14}$ \\
0.4 & 1.48 & 1.48 & 0 & $1 \times 10^{-14}$ \\
0.6 & 2.08 & 2.08 & 0 & $2 \times 10^{-14}$ \\
0.8 & 2.92 & 2.92 & 0 & $1 \times 10^{-14}$ \\
1.0 & 4 & 4 & 0 & $1 \times 10^{-14}$ \\
\hline$s$ & Exact & Proposed & Absolute errors & Absolute errors \\
& $u_{2}(s)$ & $u_{2,3}(s)$ & $e_{2,3}(s)$ & $3.1 \times 10^{-14}$ \\
0 & -1 & -1 & 0 & $2.7 \times 10^{-14}$ \\
0.2 & -0.592 & -0.592 & 0 & $2.4 \times 10^{-14}$ \\
0.4 & -0.136 & -0.136 & $5.55112 \times 10^{-17}$ & $2.3 \times 10^{-14}$ \\
0.6 & 0.416 & 0.416 & $1.11022 \times 10^{-16}$ & $2.0 \times 10^{-14}$ \\
0.8 & 1.112 & 1.112 & 0 & $2.0 \times 10^{-14}$ \\
1.0 & 2 & 2 & 0 & 2.04 \\
\hline
\end{tabular}




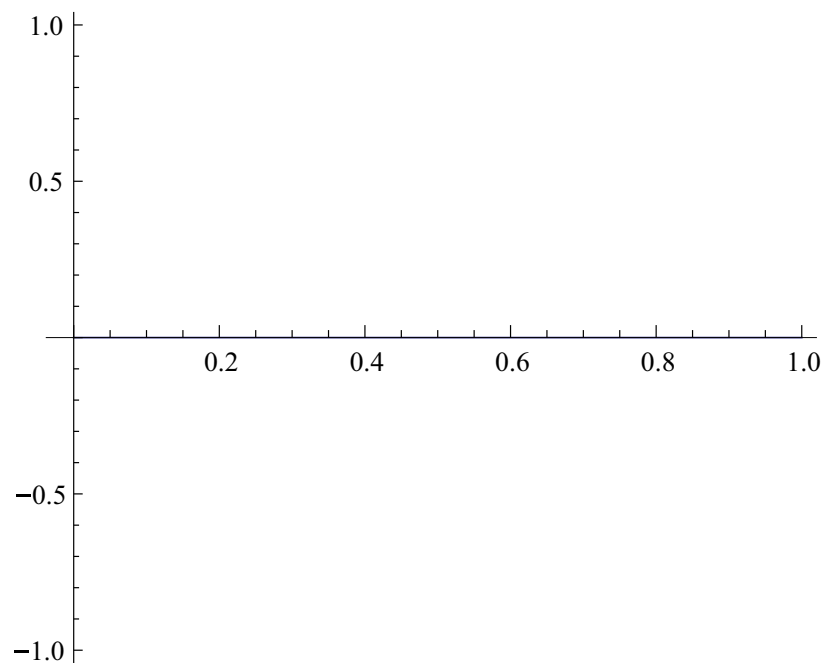

Fig. 1 Absolute errors $e_{1,3}(s)$ for $N=3$

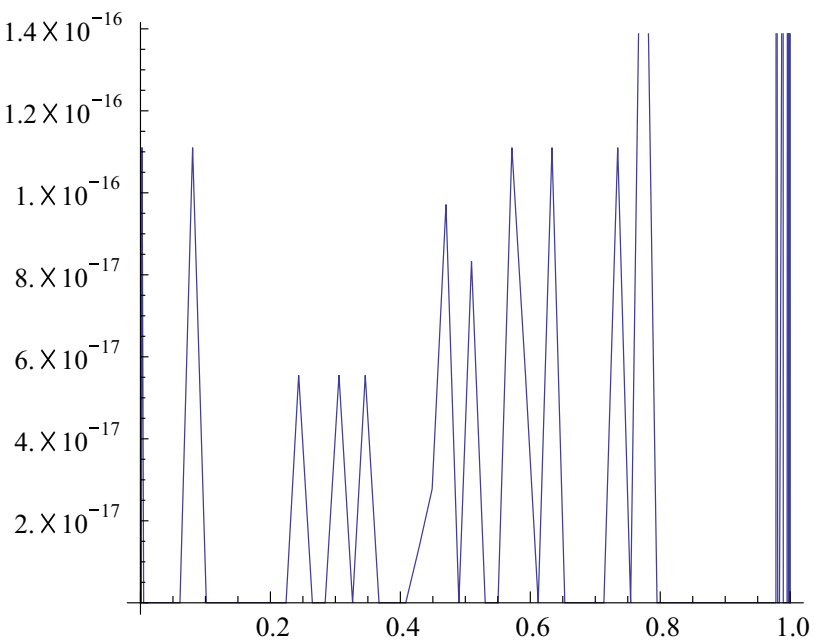

Fig. 2 Absolute errors $e_{2,3}(s)$ for $N=3$

$u_{1}^{(1)}(s)=\frac{5 s}{3}+\int_{0}^{1}\left\{\frac{s}{2} u_{1}(t)+\frac{s}{3} u_{2}(t)\right\} \mathrm{d} t$

$u_{2}^{(1)}(s)=\frac{7}{6}+\int_{0}^{1}\left\{u_{1}(t)-u_{2}(t)\right\} \mathrm{d} t, 0 \leq s \leq 1$, $u_{1}(0)=0, \quad u_{2}(0)=0$.

The analytical solution is $u_{1}(s)=s^{2}$ and $u_{2}(s)=s$.

Solving the system of equations for $N=2$ by following the procedure stated above yields the approximate solutions $u_{1,2}(s)=s^{2}$ and $u_{2,2}(s)=s$ which are exactly the same as the analytical one. Numerical results obtained by the proposed technique are shown in Table 3, while the comparison of the maximum absolute errors of the proposed technique with Adomian decomposition method [25] is $\quad\left\|e\left(u_{1,2}\right)\right\|_{\infty}=0, \quad\left\|e\left(u_{2,2}\right)\right\|_{\infty}=0 \quad$ and $\left\|e\left(u_{1,20}\right)\right\|_{\infty}=0.18 \times 10^{-6}, \quad\left\|e\left(u_{2,20}\right)\right\|_{\infty}=0.123 \times 10^{-5}$, respectively.

Example 4 Consider the system of Fredholm IDEs, as

$$
\begin{aligned}
& u_{1}^{(2)}(s)-s u_{2}^{(1)}(s)+2 s u_{1}(s)=2 s^{3}-\frac{37 s^{2}}{12}+\frac{320 s}{60}+2 \\
& +\int_{0}^{1}\left\{s^{2} t u_{1}(t)-s t^{2} u_{2}(t)\right\} \mathrm{d} t-2 s u_{1}^{(1)}(s)+u_{2}^{(2)}(s)+u_{2}(s) \\
& =-5 s^{2}-\frac{109 s}{30}-1-\int_{0}^{1}\left\{s t u_{1}(t)+s t^{3} u_{2}(t)\right\} \mathrm{d} t \\
& 0 \leq s \leq 1
\end{aligned}
$$

subject to the following boundary conditions

$$
u_{1}(0)=3, \quad u_{1}(1)=2, \quad u_{2}(0)=1, \quad u_{2}(1)=1 .
$$

The analytical solution is $u_{1}(s)=s^{2}-2 s+3$ and $u_{2}(s)=-s^{2}+s+1$.

Solving the system of equations for $N=2$ by following the procedure stated above yields the approximate solutions $u_{1,2}(s)=s^{2}-2 s+3$ and $u_{2,2}(s)=-s^{2}+s+1$ which are exactly the same as the analytical one. Numerical results obtained by the proposed technique are shown in Table 4.

Example 5 Consider the system of Fredholm IDEs, as

subject to the following initial conditions

Table 2 Numerical results for Example 2

\begin{tabular}{llllccc}
\hline$s$ & $\begin{array}{l}\text { Exact } \\
u_{1}(s)\end{array}$ & $\begin{array}{l}\text { Proposed } \\
u_{1,3}(s)\end{array}$ & $\begin{array}{l}\text { Absolute errors } \\
e_{1,3}(s)\end{array}$ & $\begin{array}{l}\text { Exact } \\
u_{2}(s)\end{array}$ & $\begin{array}{l}\text { Proposed } \\
u_{2,3}(s)\end{array}$ & $\begin{array}{l}\text { Absolute errors } \\
e_{2,3}(s)\end{array}$ \\
\hline 0 & 0 & 0 & 0 & 0 & 0 & 0 \\
0.2 & 0.086667 & 0.086667 & 0 & -0.092 & -0.092 & 0 \\
0.4 & 0.213333 & 0.213333 & 0 & -0.136 & -0.136 & 0 \\
0.6 & 0.38 & 0.38 & 0 & -0.084 & -0.084 & 0 \\
0.8 & 0.586667 & 0.586667 & 0 & 0.112 & 0.112 & 0 \\
1.0 & 0.833333 & 0.833333 & 0 & 0.5 & 0.5 & 0 \\
\hline
\end{tabular}


Table 3 Numerical results for Example 3

Table 4 Numerical results for Example 4

Table 5 Numerical results for Example 5

\begin{tabular}{lllllll}
\hline$s$ & $\begin{array}{l}\text { Exact } \\
u_{1}(s)\end{array}$ & $\begin{array}{l}\text { Proposed } \\
u_{1,2}(s)\end{array}$ & $\begin{array}{l}\text { Absolute errors } \\
e_{1,2}(s)\end{array}$ & $\begin{array}{l}\text { Exact } \\
u_{2}(s)\end{array}$ & $\begin{array}{l}\text { Proposed } \\
u_{2,2}(s)\end{array}$ & $\begin{array}{l}\text { Absolute errors } \\
e_{2,2}(s)\end{array}$ \\
\hline 0 & 0 & 0 & 0 & 0 & 0 & 0 \\
0.2 & 0.04 & 0.04 & 0 & 0.2 & 0.2 & 0 \\
0.4 & 0.16 & 0.16 & 0 & 0.4 & 0.4 & 0 \\
0.6 & 0.36 & 0.36 & 0 & 0.6 & 0.6 & 0 \\
0.8 & 0.64 & 0.64 & 0 & 0.8 & 0.8 & 0 \\
1.0 & 1 & 1 & 0 & 1 & 1 & 0 \\
\hline
\end{tabular}

\begin{tabular}{lllllll}
\hline$s$ & $\begin{array}{l}\text { Exact } \\
u_{1}(s)\end{array}$ & $\begin{array}{l}\text { Proposed } \\
u_{1,2}(s)\end{array}$ & $\begin{array}{l}\text { Proposed errors } \\
e_{1,2}(s)\end{array}$ & $\begin{array}{l}\text { Exact } \\
u_{2}(s)\end{array}$ & $\begin{array}{l}\text { Proposed } \\
u_{2,2}(s)\end{array}$ & $\begin{array}{l}\text { Proposed errors } \\
e_{2,2}(s)\end{array}$ \\
\hline 0 & 3 & 3 & 0 & 1 & 1 & 0 \\
0.2 & 2.64 & 2.64 & 0 & 1.16 & 1.16 & 0 \\
0.4 & 2.36 & 2.36 & $4.44089 \times 10^{-16}$ & 1.24 & 1.24 & 0 \\
0.6 & 2.16 & 2.16 & 0 & 1.24 & 1.24 & 0 \\
0.8 & 2.04 & 2.04 & 0 & 1.16 & 1.16 & 0 \\
1.0 & 2 & 2 & 0 & 1 & 1 & 0 \\
\hline
\end{tabular}

\begin{tabular}{|c|c|c|c|c|}
\hline$s$ & $\begin{array}{l}\text { Exact } \\
u_{1}(s)\end{array}$ & $\begin{array}{l}\text { Proposed } \\
u_{1,3}(s)\end{array}$ & $\begin{array}{l}\text { Proposed } \\
u_{1,4}(s)\end{array}$ & $\begin{array}{l}\text { Proposed } \\
u_{1,5}(s)\end{array}$ \\
\hline 0 & 0 & $-1.9984 \times 10^{-15}$ & $-1.33227 \times 10^{-15}$ & $2.3892 \times 10^{-13}$ \\
\hline 0.2 & 0.198669 & 0.19869 & 0.198517 & 0.198669 \\
\hline 0.4 & 0.389418 & 0.389516 & 0.388638 & 0.389419 \\
\hline 0.6 & 0.564642 & 0.564618 & 0.563349 & 0.564646 \\
\hline 0.8 & 0.717356 & 0.716132 & 0.717141 & 0.717369 \\
\hline 1.0 & 0.841471 & 0.836195 & 0.846011 & 0.841529 \\
\hline$s$ & $\begin{array}{l}\text { Exact } \\
u_{2}(s)\end{array}$ & $\begin{array}{l}\text { Proposed } \\
u_{2,3}(s)\end{array}$ & $\begin{array}{l}\text { Proposed } \\
u_{2,4}(s)\end{array}$ & $\begin{array}{l}\text { Proposed } \\
u_{2,5}(s)\end{array}$ \\
\hline 0 & 1 & 1 & 1 & 1 \\
\hline 0.2 & 0.980067 & 0.980214 & 0.978639 & 0.980066 \\
\hline 0.4 & 0.921061 & 0.921711 & 0.917059 & 0.92106 \\
\hline 0.6 & 0.825336 & 0.825775 & 0.83689 & 0.825337 \\
\hline 0.8 & 0.696707 & 0.693689 & 0.783602 & 0.696719 \\
\hline 1.0 & 0.540302 & 0.526737 & 0.8265 & 0.540404 \\
\hline
\end{tabular}

Table 6 Maximum absolute errors for Example 5

\begin{tabular}{llll}
\hline$N$ & 3 & 4 & 5 \\
\hline$e_{1, N}$ & $5.27579 \times 10^{-3}$ & $4.53959 \times 10^{-3}$ & $5.80152 \times 10^{-5}$ \\
$e_{2, N}$ & $1.35651 \times 10^{-2}$ & $2.86198 \times 10^{-1}$ & $1.01691 \times 10^{-4}$ \\
\hline
\end{tabular}

$$
\begin{aligned}
& u_{1}^{(2)}(s)-s u_{2}^{(1)}(s)-u_{1}(s)=(s-2) \sin (s) \\
& \quad+\int_{0}^{1}\left\{s \cos (t) u_{1}(t)-s \sin (t) u_{2}(t)\right\} \mathrm{d} t \\
& \quad-2 s u_{1}^{(1)}(s)+u_{2}^{(2)}(s)+u_{2}(s)=-2 s \cos (s) \\
& \quad+\int_{0}^{1}\left\{\sin (s) \cos (t) u_{1}(t)-\sin (s) \sin (t) u_{2}(t)\right\} \mathrm{d} t \\
& 0 \leq s \leq 1
\end{aligned}
$$

subject to the following initial conditions 


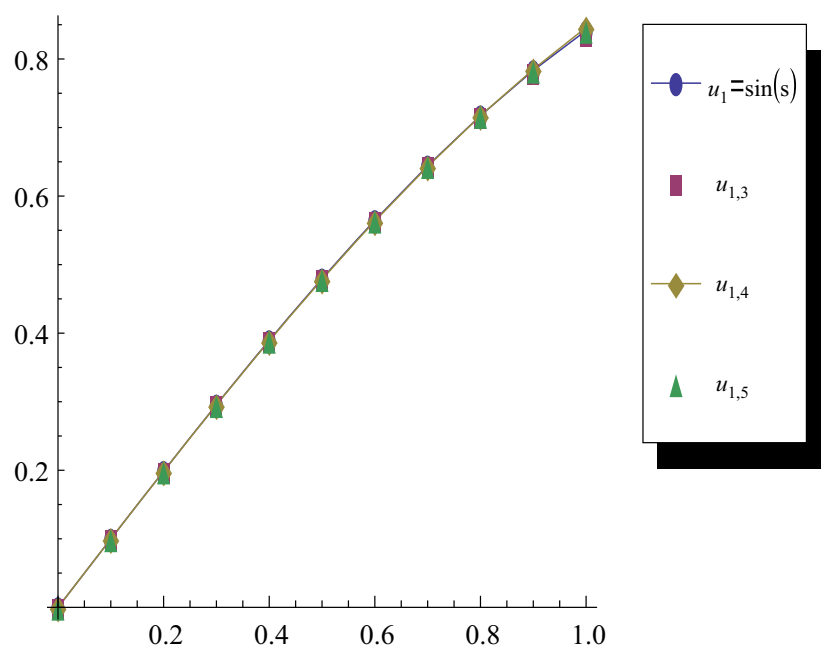

Fig. 3 Comparison of $u_{1}(s)$ and $u_{1, N}$ for $N=3,4,5$

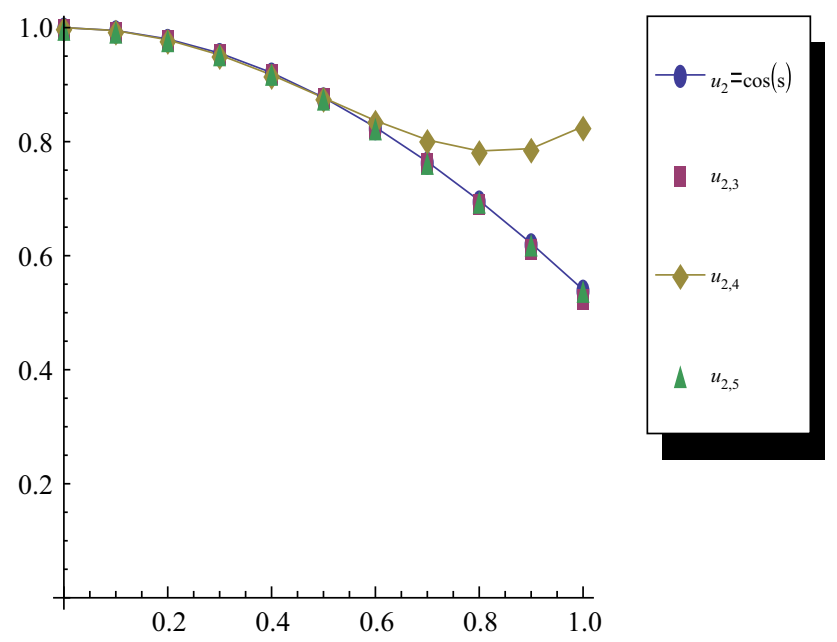

Fig. 4 Comparison of $u_{2}(s)$ and $u_{2, N}$ for $N=3,4,5$

$u_{1}(0)=0, \quad u_{1}^{(1)}(0)=1, \quad u_{2}(0)=1, \quad u_{2}^{(1)}(0)=0$.

The analytical solution is $u_{1}(s)=\sin (s)$ and $u_{2}(s)=\cos (s)$.

Solving the system of equations for $N=3,4,5$ by following the procedure stated above, yields the approximate solutions:

$$
\begin{aligned}
u_{1,3}(s)= & -1.9984 \times 10^{-15}+1 s-8.88178 \times 10^{-16} s^{2} \\
& -0.163805 s^{3}, \\
u_{2,3}(s)= & 1-3.33067 \times 10^{-16} s-0.5 s^{2}+0.0267372 s^{3}, \\
& \text { and } \\
u_{1,4}(s)= & -1.33227 \times 10^{-15}+1 s-1.33227 \times 10^{-14} s^{2} \\
& -0.193214 s^{3}+0.0392248 s^{4},
\end{aligned}
$$

$$
\begin{aligned}
u_{2,4}(s)= & 1+8.52651 \times 10^{-14} s-0.5 s^{2}-0.294265 s^{3} \\
& +0.620765 s^{4} \\
& \text { also } \\
u_{1,5}(s)= & 2.3892 \times 10^{-13}+1 s+4.01457 \times 10^{-13} s^{2} \\
& -0.166728 s^{3}+0.000337574 s^{4} \\
& +0.00791926 s^{5}, \\
u_{2,5}(s)= & 1 .-8.08242 \times 10^{-13} s-0.5 s^{2}-0.00030213 s^{3} \\
& +0.0431559 s^{4}-0.00244974 s^{5} .
\end{aligned}
$$

Numerical results are summarized in Tables 5, while 6 shows the maximum absolute errors for Example 5. Comparison of exact and proposed solutions is shown in Figs. 3 and 4 for $N=3,4$ and 5, respectively.

\section{Conclusion}

In this paper, Laguerre operational matrix approach has been manipulated to solve the system of linear Fredholm IDEs. The scheme converted the system of IDEs, using Laguerre operational matrices, to a matrix equation that can be solved by any suitable method. Comparison of the results with other methods such as Tau method [21] and Adomian decomposition method (ADM) [25] reveals that the Laguerre approach has more accuracy. In addition, to get the best approximating solution of the system, the truncation limit $N$ must be chosen large enough. It is also to be mentioned that the method is efficient to determine the solution in closed form, as well.

\section{Compliance with ethical standards}

Conflict of interest All the authors declare that they have no conflict of interest.

Open Access This article is distributed under the terms of the Creative Commons Attribution 4.0 International License (http://creative commons.org/licenses/by/4.0/), which permits unrestricted use, distribution, and reproduction in any medium, provided you give appropriate credit to the original author(s) and the source, provide a link to the Creative Commons license, and indicate if changes were made.

\section{References}

1. Kurt, N., Çevik, M.: Polynomial solution of the single degree of freedom system by Taylor matrix method. Mech. Res. Commun. 35, 530-536 (2008)

2. Kurt, N., Sezer, M.: Polynomial solution of high-order linear Fredholm integro-differential equations with constant coefficients. J. Frankl. Inst. 345, 839-850 (2008)

3. Coulaud, O., Funaro, D., Kavian, O.: Laguerre spectral approximation of elliptic problems in exterior domains. Comput. Methods Appl. Mech. Eng. 80, 451-458 (1990) 
4. Abd-Elhameed, W.M., Youssri, Y.H., Doha, E.H.: A novel operational matrix method based on shifted Legendre polynomials for solving second-order boundary value problems involving singular, singularly perturbed and Bratu-type equations. Math. Sci. 9, 93-102 (2015)

5. Oğuz, C., Sezer, M.: Chebyshev collocation method for a class of mixed functional integro-differential equations. Appl. Math. Comput. 259, 943-954 (2015)

6. Erfanian, M., Zeidabadi, H.: Using of Bernstein spectral Galerkin method for solving of weakly singular Volterra-Fedholm integral equations. Math. Sci. (2018). https://doi.org/10.1007/s40096-0180249-1

7. Mavromatis, H.A., Alassar, R.S.: Two new associated Laguerre integral results. Appl. Math. Lett. 14, 903-905 (2001)

8. Ezz-Eldien, S.S., Doha, E.H.: Fast and precise spectral method for solving pantograph type Volterra integro-differential equations. Numer. Algorithm (2018). https://doi.org/10.1007/s11075018-0535-x

9. Ezz-Eldien, S.S.: On solving systems of multi-pantograph equations via spectral tau method. Appl. Math. Comput. 321, 63-73 (2018)

10. Bhrawy, A.H., Abdelkawy, M.A., Ezz-Eldien, S.S.: Efficient spectral collocation algorithm for a two-sided space fractional Boussinesq equation with non-local conditions. Mediterr. J. Math. 13, 2483-2506 (2016)

11. Bhrawy, A.H., Ezz-Eldien, S.S.: A new Legendre operational technique for delay fractional optimal control problems. Calcolo 53, 521-543 (2016)

12. Ezz-Eldien, S.S.: New quadrature approach based on operational matrix for solving a class of fractional variational problems. J. Comput. Phys. 317, 362-381 (2016)

13. Ezz-Eldien, S.S., El-Kalaawy, A.A.: Numerical simulation and convergence analysis of fractional optimization problems with right-sided Caputo fractional derivative. J. Comput. Nonlinear Dyn. 13(1), 011010 (2018). (8 pages)

14. Xu, L., He, J.H., Liu, Y.: Electrospun nanoporous spheres with Chinese drug. Int. J. Nonlinear Sci. Numer. Simul. 8(2), 199-202 (2007)

15. Wang, H., Fu, H.M., Zhang, H.F.: A practical thermodynamic method to calculate the best glass-forming composition for bulk metallic glasses. Int. J. Nonlinear Sci. Numer. Simul. 8(2), 171-178 (2008)

16. Sun, F.Z., Gao, M., Lei, S.H.: The fractal dimension of the fractal model of dropwise condensation and its experimental study. Int. J. Nonlinear Sci. Numer. Simul. 8(2), 211-22 (2007)

17. Bo, T.L., Xie, L., Zheng, X.L.: Numerical approach to wind ripple in desert. Int. J. Nonlinear Sci. Numer. Simul. 8(2), 223-228 (2007)

18. Bellomo, N., Firmani, B., Guerri, L.: Bifurcation analysis for a nonlinear system of integro-differential equations modelling tumor-immune cells competition. Appl. Math. Lett. 12, 39-44 (1999)

19. Wazwaz, A.M.: The existence of noise terms for systems of inhomogeneous differential and integral equations. Appl. Math. Comput. 146, 81-92 (2003)

20. Singh, R., Wazwaz, A.M.: Numerical solutions of fourth-order Volterra integro-differential equations by the Greens function and decomposition method. Math. Sci. 10, 159-166 (2016)

21. Pour-Mahmoud, J., Rahimi-Ardabili, M.Y., Shahmorad, S.: Numerical solution of the system of Fredholm integro-differential equations by the Tau method. Appl. Math. Comput. 168, 465-478 (2005)
22. Mirzaee, F., Hoseini, S.F.: Solving systems of linear Fredholm integro-differential equations with Fibonacci polynomials. Ain Shams Eng. J. 5, 271-283 (2014)

23. Yuzba, S., Sahin, N., Sezer, M.: Numerical solutions of systems of linear Fredholm integro-differential equations with Bessel polynomial bases. Comput. Math. Appl. 61, 3079-3096 (2011)

24. Mollaoğlu, T., Sezer, M.: A numerical approach with residual error estimation for evolution high-order linear differential-difference equations by using Gegenbauer polynomials. Sains Malays. 46, 335-347 (2017)

25. Khanain, M., Davari, A.: Solution of system of Fredholm integrodifferential equations by Adomian decomposition method. Aust. J. Basic Appl. Sci. 5(12), 2356-2361 (2011)

26. Rabbani, M., Zarali, B.: Solution of Fredholm integro-differential equations system by modified decomposition method. J. Math. Comput. Sci. 5(4), 258-264 (2012)

27. Maleknejad, K., Tavassoli, M.K.: Solving linear integro-differential equation system by Galerkin methods with hybrid functions. Appl. Math. Comput. 159, 603-612 (2004)

28. Elahi, Z., Akram, G., Siddiqi, S.S.: Numerical solution for solving special eighth-order linear boundary value problems using Legendre Galerkin method. Math. Sci. 10, 201-209 (2016)

29. Elahi, Z., Akram, G., Shahid, S.: Siddiqi, Numerical solutions for solving special tenth-order linear boundary value problems using Legendre Galerkin method. Math. Sci. Lett. 7, 27-35 (2018)

30. Nouri, K., Torkzadeh, L., Mohammadian, S.: Hybrid Legendre functions to solve differential equations with fractional derivatives. Math. Sci. (2018). https://doi.org/10.1007/s40096-0180251-7

31. Arikoglu, A., Ozkol, I.: Solutions of integral and integro-differential equation systems by using differential transform method. Comput. Math. Appl. 56, 2411-2417 (2008)

32. Maleknejad, K., Safdari, H., Nouri, M.: Numerical solution of an integral equations system of the first kind by using an operational matrix with block pulse functions. Int. J. Syst. Sci. 42(1), 195-199 (2011)

33. Gülsu, M., Sezer, M.: Taylor collocation method for solution of systems of high-order linear Fredholm Volterra integro-differential equations. Int. J. Comput. Math. 83, 429-448 (2006)

34. Bahşi, M.M., Bahşi, A.K., Çevik, M., Sezer, M.: Improved Jacobi matrix method for the numerical solution of Fredholm integrodifferential-difference equations. Math. Sci. 10, 83-93 (2016)

35. Balci, M.A., Sezer, M.: Hybrid Euler-Taylor matrix method for solving of generalized linear Fredholm integro-differential-difference equations. Appl. Math. Comput. 273, 33-41 (2016)

36. Yalçinbaş, S., Sezer, M., Sorkun, H.H.: Legendre polynomial solutions of high-order linear Fredholm integro-differential equations. Appl. Math. Comput. 210, 334-349 (2009)

37. Maleknejad, K., Mahmoudi, Y.: Taylor polynomial solutions of high-order nonlinear Volterra-Fredholm integro-differential equation. Appl. Math. Comput. 145, 641-653 (2003)

38. Bayku, N., Sezer, M.: Hybrid Taylor-Lucas colocation method for numerical solution of high-order pantograph type delay differential equations with variables delays. Appl. Math. Inf. Sci. 11, 1795-1801 (2017)

Publisher's Note Springer Nature remains neutral with regard to jurisdictional claims in published maps and institutional affiliations. 